\title{
Influence of Swirl Intensity and Blended Methane- Hydrogen on Gas Turbine Combustion Characteristics
}

\author{
Mohammed W. Eldeen \\ Department of Mechanical Engineering \\ Arab Academy for Science and \\ Technology Alexandria, Egypt
}

\author{
Mohammed A. Taemah \\ Department of Mechanical Engineering \\ Arab Academy for Science and \\ Technology Alexandria, Egypt
}

\author{
Wael M. El-Maghalany \\ Department of Mechanical \\ Engineering Alexandria University \\ Alexandria, Egypt
}

\begin{abstract}
This paper presents numerical investigation of the effect of swirl number and hydrogen addition on combustion characteristic such as temperature, and major species concentrations in a natural gas turbulent diffusion flame which is essential occurrence in a gas turbine combustion system. The analysis is performed numerically using the computational fluid dynamics code ANSYS FLUENT. The study is conducted in two sections, the first section, a three RANS turbulence models are used to close the Reynolds stresses, coupled with steady laminar flamelet model as combustion model and p-1 as radiation model. The obtained numerical results are validated and compared with the high quality experimental data base SM1 flame Sydney swirl burner available from Sendia national laboratories, the university of Sydney. The results also demonstrated that the RSM model could provide a reasonably accurate prediction data that is in consistent with experimental measurements at most locations. The second part, the RSM is utilized to investigate the effect of swirl number and blended methane-hydrogen on combustion characteristics.
\end{abstract}

Keywords: Turbulent diffusion flames Turbulence models, swirl flow, RANS, CFD, Sydney swirl burner Laminar flamelet model

\section{INTRODUCTION}

Studying turbulent combustion remains an important research subject of interest for engineers due to its widely used in engineering applications such as gas turbine combustors, furnaces and burners [1]. As these applications are required to provide high performance (i.e. efficiency, reliability and stability while meeting strict low emissions requirements), enhancements are often necessary. Introducing swirl in turbulent combustion offers an advantage in terms of flame stabilization, mixing enhancement and pollutant diminution. Swirl can be induced geometrically through vane swirls or helical inserts within a nozzle, mechanically through rotation or aerodynamically by tangential injection into a stream wise flow as this study, swirling can be defined as the ratio of the tangential momentum flux to the axial momentum flux [2]. Centrifugal forces present in the swirl flow apply a significant adverse pressure gradient in axial direction which accompanied by decrease in velocity, this in turn causes a vortex type recirculation zone in the central region of the jet, this vortex type known as vortex break down acts as storage of heat and mass [3]. As the flow reversed because of recirculation some of the well mixed combustion products return to the flame fronts to mix with the fresh combustion mixture that ensures high combustion efficiency and flame stability. There are limitations related to the usage of fossil fuels due to pollutant effects and fossil fuels are running out all over the world. Blended hydrocarbon-H2 fuel is one of the challenging, it was demonstrated that the use of certain proportion of hydrogen with hydrocarbons improve the performance of combustion (improved stability, reduced pollution, etc.). Extensive experimental investigations have been performed to study turbulent diffusion flames to understand the flow behavior and to discover the influence of many parameters on the flow field structure and burning characteristics of the flames. Because of scale, limited access, expenses and other practical considerations of real combustors, recent experimental investigation focuses on laboratory scale burners [4]. One of the well-designed experimental burner is the laboratory-swirl burners which are representative of real combustors but designed with simpler, well-defined, and optically accessible configurations to enable detailed data acquisition and facilitate modelling. The importance of numerical simulation for turbulent/combustion interaction stems mainly from the intensive cost and time being consumed when an experimental investigation is conducted. Computational fluid dynamics provide a promising numerical tool that have been used to investigate the swirling diffusion flames which pose a challenging problem for CFD simulation, this mainly due to its additional complexity caused by large density variation, turbulent modified, chemical reaction rates and radiation heat transfer. Methods based on Reynolds Averaged Navier-Stokes equations (RANS) have been widely used because of their quick turn-around times. The LES technique can be deemed as a powerful numerical tool which can provide consistent and excellent predictions compared to experimental measurements. Such model can be also used even in cases where there is no previous experimental data exist. However, they are relatively expensive in terms of computer resources and time requirements compared to the classical models based on RANS approach. While in practice engineering applications, engineers are not usually interested in accurate data that are closely matching the real measurements. Instead, moderate predictions with reasonable computational costs such as those models based on RANS technique would be fairly accepted. 


\section{MATHEMATICAL MODELLING AND NUMERICAL METHOD}

A. Reynolds Averaged-Navier Stokes (RANS)

The governing equations describing the turbulent combustion flow can be derived from the principles of conservation of mass, momentum, species and energy.

- Mass conservation (continuity)

$$
\frac{\partial \overline{\rho u_{j}}}{\partial x_{j}}=\mathbf{0}
$$

- Momentum conservation:

$$
\begin{aligned}
& \frac{\partial\left({\overline{\rho u_{i}}}_{\bar{u}}\right)}{\partial x_{j}}=-\frac{\partial \bar{P}}{\partial x_{i}}+\frac{\partial\left(\bar{t}_{i j}+\bar{\tau}_{i j}\right)}{\partial x_{j}} \\
& t_{i j}=\mu\left[\left(\frac{\partial u_{i}}{\partial x_{j}}+\frac{\partial u_{j}}{\partial u_{i}}\right)-\frac{2}{3} \delta_{i j}\left(\frac{\partial u_{k}}{\partial x_{k}}\right)\right] \\
& \tau_{i j}=-\rho u i u j=\mu_{t}\left[\left(\frac{\partial u_{i}}{\partial x_{j}}+\frac{\partial u_{j}}{\partial u_{i}}\right)-\frac{2}{3} \delta_{i j}\left(\frac{\partial u_{k}}{\partial x_{k}}\right)\right]-\frac{2}{3}\left(\overline{\rho k \delta_{\imath \jmath}}\right) \\
& \mu_{t}=C_{\mu} \rho \frac{k^{2}}{\varepsilon} \\
& k=\frac{1}{2} \overline{u_{\imath} u_{\imath}} \\
& \varepsilon=v \frac{\overline{\partial u_{i}}}{\partial x_{j}} \frac{\partial u_{i}}{\partial x_{j}}
\end{aligned}
$$

\section{B. Turbulence modelling}

\section{- Two equations models}

The standard $\mathbf{k}-\boldsymbol{\varepsilon}$

$\frac{\partial}{\partial x_{j}}\left(\rho k u_{j}\right)=\frac{\partial}{\partial x_{j}}\left(\left(\mu+\frac{\mu_{t}}{\sigma_{k}}\right) \frac{\partial k}{\partial x_{j}}\right)+2 \mu_{t} s_{i j} S_{i j}-\rho \varepsilon$

$$
\frac{\partial \varepsilon}{\partial x_{j}}\left(\rho \varepsilon u_{j}\right)=\frac{\partial}{\partial x_{j}}\left(\left(\mu+\frac{\mu_{t}}{\sigma_{\varepsilon}}\right) \frac{\partial \varepsilon}{\partial x_{j}}\right)+2 C_{1 \varepsilon} \frac{\varepsilon}{k} \mu_{t} s_{i j} s_{i j}-C_{2 \varepsilon} \rho \frac{\varepsilon^{2}}{k}
$$

\section{The modified $\mathbf{k}-\boldsymbol{\varepsilon}$}

This model has the same equations of standard k- $\varepsilon$ but Dally et al. [5] modified the constant $C_{1 \varepsilon}$ in the dissipation transport equation of standard $\mathrm{k}-\varepsilon$, which gives better predictions of the flow and mixing fields, the $C_{1 \varepsilon}$ constant $=1.6$ instead of 1.44 .

- Reynolds stress model (RSM)

This approach is also known as a second order moment closure [6]. It is believed that solving the transport equations of Reynolds stresses produces better results than those produced by the two equation models. The major advantage of the RSM over the two equation models is its capability of representing the highly anisotropic nature of the flow. The model also accounts for the streamlines curvature.

The transport equation for the Reynolds stresses $-\rho \overline{u_{l} u_{\jmath}}$ can be expressed as:

$$
\begin{aligned}
\frac{\partial}{\partial x_{k}}\left(\rho u_{k} \overline{u_{i}^{\prime} u_{j}^{\prime}}\right) & \\
= & \frac{\partial}{\partial x_{k}}\left(\frac{\mu_{t}}{\sigma_{k}} \frac{\partial \overline{u_{\imath}^{\prime} u_{j}^{\prime}}}{\partial x_{k}}\right)+\frac{\partial}{\partial x_{k}}\left[\mu \frac{\partial}{\partial x_{k}}\left(\overline{u_{i}^{\prime} u_{j}^{\prime}}\right)\right] \\
& -\rho\left(\overline{u_{\imath}^{\prime} u_{j}^{\prime}} \frac{\partial u_{j}}{\partial x_{k}}+\overline{u_{j}^{\prime} u_{i}^{\prime}} \frac{\partial u_{i}}{\partial x_{k}}\right)+\Phi_{i j}-\frac{2}{3} \delta_{i j} \rho \varepsilon
\end{aligned}
$$

The term on the left side of the above equation represents the convective term, and the terms on the right-hand side represent the turbulent diffusion, molecular diffusion, stress production $\left(P_{i j}\right)$, pressure strain $\left(\Phi_{i j}\right)$ and the dissipation, respectively.

$$
\begin{aligned}
& \Phi_{i j}=\Phi_{i j, 1}+\Phi_{i j, 2}+\Phi_{i j, w} \\
& \text { Where } \\
& \Phi_{i j, 1}=-C_{1} \rho \frac{\varepsilon}{k}\left[\overline{u_{\imath}^{\prime} u_{j}^{\prime}}-\frac{2}{3} \delta_{i j} k\right] \\
& \Phi_{\mathrm{ij}, 2}=-\mathrm{C}_{2}\left[\left(\mathbf{P}_{\mathrm{ij}}-\mathrm{C}_{\mathrm{ij}}\right)-\frac{1}{3} \delta_{\mathrm{ij}}\left(\mathbf{P}_{\mathrm{kk}}-\mathrm{C}_{\mathrm{kk}}\right)\right] \\
& \Phi_{i j, w}=C_{1}^{\prime} \frac{\varepsilon}{k}\left(\overline{u_{k}^{\prime} u_{m}^{\prime}} n_{k} n_{m} \delta_{i j}-\frac{3}{2} \overline{u_{1}^{\prime} \mathbf{u}_{k}^{\prime}} n_{j} n_{k}-\frac{3}{2} \overline{u_{i}^{\prime} \mathbf{u}_{k}^{\prime}} n_{j} n_{k}\right) \frac{k^{3 / 2}}{C_{l \varepsilon d}} \\
& +\mathrm{C}_{2}^{\prime}\left(\phi_{\mathrm{km}, 2} \mathrm{n}_{\mathrm{k}} \mathrm{n}_{\mathrm{m}} \delta_{\mathrm{ij}}-\frac{3}{2} \phi_{\mathrm{ik}, 2} \mathrm{n}_{\mathrm{j}} \mathrm{n}_{\mathrm{k}}\right. \\
& \left.-\frac{3}{2} \phi_{j k, 2} n_{j} n_{k}\right) \frac{k^{3 / 2}}{C_{2 \varepsilon d}}
\end{aligned}
$$

The model of the dissipation rate term $\varepsilon$ is the same as in the $\mathrm{k}-\varepsilon$ model

Where, $\mathbf{c}_{\mathbf{l}}=\mathbf{c}_{\boldsymbol{\mu}}^{\mathbf{3} / \mathbf{4}} / \mathbf{\kappa}$, and $\kappa$ is the von Karman constant $=$ 0.4187

The model constants, which are determined from experiments, are shown in Table1.

TABLE 1. The models' coefficients,

\begin{tabular}{|c|c|c|c|c|c|c|c|c|c|}
\hline & $C_{\mu}$ & \multicolumn{2}{|c|}{$C_{1 \varepsilon}$} & \multicolumn{2}{c|}{$C_{2 \varepsilon}$} & \multicolumn{2}{|c|}{$\sigma_{k}$} & \multicolumn{2}{|c|}{$\sigma_{\varepsilon}$} \\
\hline Standard k- $-\varepsilon$ & 0.09 & \multicolumn{2}{|c|}{1.44} & \multicolumn{2}{|c|}{1.92} & \multicolumn{2}{|c|}{1} & \multicolumn{2}{|c|}{1.3} \\
\hline Modified k- $-\varepsilon$ & 0.09 & \multicolumn{2}{|c|}{1.6} & \multicolumn{2}{|c|}{1.92} & \multicolumn{2}{|c|}{1.3} \\
\hline RSM & $C_{\mu}$ & $C_{1}$ & $C_{2}$ & $C_{1}^{\prime}$ & $C_{2}^{\prime}$ & $C_{1 \varepsilon}$ & $C_{2 \varepsilon}$ & $\sigma_{k}$ & $\sigma_{\varepsilon}$ \\
\hline & 0.09 & 1.8 & 0.6 & 0.5 & 0.3 & 1.44 & 1.92 & 1 & 1.3 \\
\hline
\end{tabular}

\section{Turbulent Combustion model}

\section{$>$ Laminar Flamelet model}

In non-premixed (diffusion) combustion the turbulent flame is considered to consist of a series of thin flames, where the combustion is assumed to occur within the thin layers such that they are embedded within the turbulent flow. These layers have a well-defined inner structure called flamelets. The laminar flamelet approach has been proposed by Peters [7]. Since The steady laminar flamelet equations, species concentrations and enthalpy balance equations are conserved, then the corresponding thermo-chemical compositions in laminar and turbulent flows satisfy the transport equations. As has been previously mentioned the turbulent nonpremixed flame can be presented by an ensemble of discrete of laminar diffusion flamelet, a formal coordinate transformation using the mixture fraction as independent variables, leads to a universal description of the flamelet properties. Transformation of transport equations from 
physical space to mixture fraction space. A simplified set of the mixture fraction space equations including species and energy is solved .

- $\quad$ Species conservation

$$
\rho \frac{\chi}{2} \frac{\partial^{2} Y_{i}}{\partial Z^{2}}+\omega_{i}=0
$$

\section{- Energy conservation}

$$
\rho \frac{\chi}{2} \frac{\partial^{2} T}{\partial Z^{2}}-\sum_{i=1}^{n} \frac{h_{i}}{C_{p}} \omega_{i}+\rho \frac{\chi}{2 C_{p}}\left[\frac{\partial C_{p}}{\partial Z}+\sum C_{p \cdot K}+\frac{\partial Y_{i}}{\partial Z}\right] \frac{\partial T}{\partial Z}=0
$$

The chemical source term is marked by $\omega_{i}$ and computed via reactions present in the chemical mechanism. An expression for the variable density flows is utilized for describing the scalar dissipation rate $\chi(Z)$ for variable density flows is calculated by the expression:

$$
\chi(Z)=\frac{a_{s}}{4 \pi} \frac{3\left(\sqrt[2]{\frac{\rho_{o}}{\rho}+1}\right)^{2}}{\sqrt[2]{\frac{\rho_{o}}{\rho}+1}} \exp \left[-2\left(\operatorname{erf}^{-1}(2 Z)\right)^{2}\right]
$$

Here $a_{s}$ is the characteristic strain rate, $e r f^{-1}$ is the inverse complementary error function and $\rho_{o}$ is the oxidizer density. In the steady flamelet model, the adiabatic flame structure is described by means of the mixture fraction and the scalar dissipation rate at stoichiometric mixture fraction. For non-adiabatic effects (heat losses due to radiation) a third parameter is needed which is the enthalpy defect $\emptyset_{H}$ ). This variable is defined as the difference between the enthalpy of the mixture for adiabatic and non-adiabatic conditions.

$$
\emptyset_{H}=\widehat{\boldsymbol{h}}-\left[\widehat{\boldsymbol{h}}_{o}+\boldsymbol{Z}\left(\widehat{\boldsymbol{h}}_{f}-\widehat{\boldsymbol{h}}_{o}\right)\right]
$$

Here $\hat{h}_{f}$ and $\hat{h}_{o}$ are the enthalpy of fuel and oxidizer streams, respectively. The enthalpy of the mixture for adiabatic conditions is considered as a linear function of the mixture fraction. It is held to be equal to the enthalpy of the oxidizer for $Z=0$ and to the enthalpy of fuel for $Z=1$. The enthalpy of the mixture for non-adiabatic conditions is computed by fixing the enthalpy defect. Hence, any quantity within the turbulent field is described by three parameters (i.e. the mixture fraction, the scalar dissipation rate and the enthalpy defect). The Favre-averaged quantities are characterized as:

$$
\bar{\varphi}=\int_{-\infty}^{\infty} \int_{0}^{\infty} \int_{0}^{1} \varphi\left(Z ; \chi_{s t} ; \emptyset_{H}\right) P\left(Z ; \chi_{s t} ; \emptyset_{H}\right) d Z d \chi_{s t} d \emptyset_{H}
$$

Statistical independence assumption is used between the variables of the overall probability density function (PDF). A beta-shape PDF is used for the mixture fraction distribution. Log-normal and Dirac delta functions are employed for the stoichiometric scalar dissipation rate and enthalpy defect respectively. Hence, equation (19) can be rewritten as:

$$
\bar{\varphi}=\int_{0}^{\infty} P\left(\chi_{s t}\right) d \chi_{s t} \int_{0}^{1} \varphi\left(Z ; \chi_{s t} ; \emptyset_{H}\right) P(Z) d Z
$$

The mean scalar dissipation rate in the mixture fraction variance equation is modelled as:

$$
\chi_{s t}=\frac{C_{x} \bar{\varepsilon} \overline{Z^{\prime 2}}}{k}
$$

Where $C_{x}=2$ and $\overline{Z^{r}}$ is the Favre mean mixture fraction variance

\section{Radiation model}

The radiation source term is computed from the optically thin radiation model [8,9]. In this method, the flame is considered optically thin such that each radiating point has an unhindered isotropic sight of the cold ambient. The source term in transport equation for enthalpy which is the radiative loss per unit volume can be obtained from the following relation:

$$
S_{h}=4 \sigma_{B}\left(T^{4}-T_{b}^{4}\right) \sum\left(p_{i} \times a_{p . i}\right)
$$

In the above equation, $\sigma_{B}$ is the Stefan-Boltzmann constant, $p_{i}$ is the partial pressure of species (i)in atmosphere and $a_{p . i}$ is the Planck mean absorption coefficient. The local flame and background temperatures are given by $\mathrm{T}$ and $T_{b}$.

\section{E. Chemical Reaction mechanism}

The present study is intended to use the well-recognized GRIMech 3.0 for methane/air reaction. The mechanism consists of 325 elementary reactions and 53 species. The temperature and species concentrations distribution are stored as a function of the distance of a flame front [10].

\section{THE NUMERICAL SOLUTION AND MODEL VALIDATION}

\section{A. Sydney swirl burner}

The current work uses the geometrical structure of Sydney university swirl burner to perform the numerical simulation study, "Fig.1," the burner is an extension of the well characterized Sydney bluff body burner for swirling flames. The burner features $60 \mathrm{~mm}$ diameter annuals for the primary swirl air which incircle a $50 \mathrm{~mm}$ diameter ceramic face bluff body with central jet tube of $3.6 \mathrm{~mm}$ diameter, the swirl is induced here aerodynamically at air stream via three tangential ports (7 mm each) located at $300 \mathrm{~mm}$ up stream of burner exit. For the purpose of well-defined boundary conditions, the burner configuration is placed in a square wind tunnel with an exit cross section of $130 \times 130 \mathrm{~mm}$. The swirl configuration used features a non-premixed flame stabilized by an upstream recirculation zone caused by a bluff body and second downstream recirculation zone induced by swirl, which greatly improve the mixing process. 


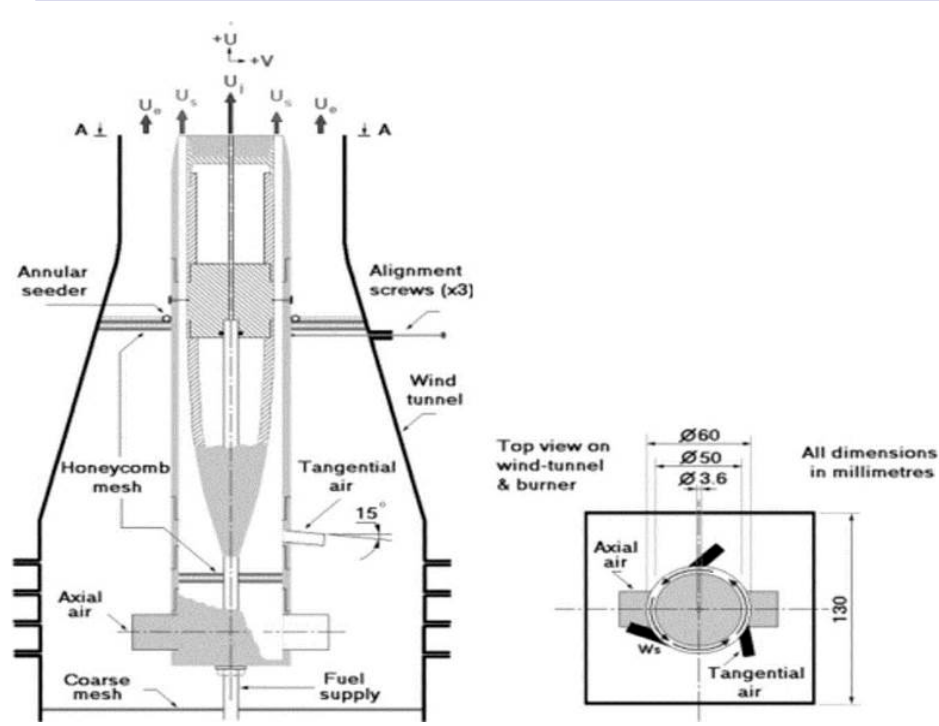

Fig. 1. Schematic diagram of Sydney swirl burner [11].

Four parameters determine the flame characteristics are: $\mathrm{Uj}$, Us, Ws and Ue. Where Uj is the central jet velocity, Us is the bulk axial velocity of the annulus, Ws is the bulk tangential velocity and Ue is the co-flow velocity of the wind tunnel. The quantity of swirl numbers Sg $=$ Ws $/ \mathrm{Us}$, the properties of the simulated flame SM1 is summarized in Table 2.

TABLE 2. Parameters of the investigated case (SM1).

\begin{tabular}{|c|c|c|c|c|c|c|c|}
\hline case & Fuel & $\mathrm{Uj}(\mathrm{m} / \mathrm{s})$ & $\mathrm{Us}(\mathrm{m} / \mathrm{s})$ & $\mathrm{Ws}(\mathrm{m} / \mathrm{s})$ & $\mathrm{Ue}(\mathrm{m} / \mathrm{s})$ & $\mathrm{Sg}$ & $\mathrm{Re}$ \\
\hline SM1 & $\mathrm{CH} 4$ & 32.7 & 38.2 & 19.1 & 20 & 0.5 & 7200 \\
\hline
\end{tabular}

To simplify the analysis, the following assumptions are made

- The reacting flow is assumed to be steady state and axisymmetric.

- The gravitational effect is neglected.

B. Simulation setup and solution strategies

- Geometrical model

For simulation purpose the burner dimension is considered exactly as in real case. Because of symmetry only half of burner is discretized on the computational domain. the 2D axisymmetric geometry and the grid generation were performed with the aid of FLUENT. "Fig. 2," represents the geometrical and grid (all dimensions are in $\mathrm{mm}$ )
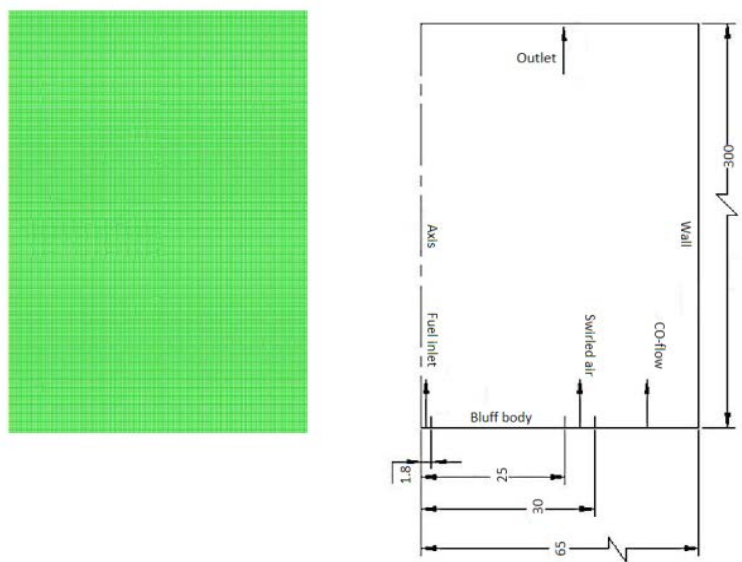

Fig. 2. schematic diagram of the geometry and mesh used in the simulation

\section{- $\quad$ Numerical Solver}

In the current research, the ANSYS FLUENT has been utilized as the computational platform. The Semi-Implicit Method for Pressure Linked Equations (SIMPLE) is applied for the pressure-velocity coupling [12]. The second order up wind is applied for the convection terms and standard method is used for the pressure discretization. The convergence criteria were set to $\mathbf{1 0}^{-\mathbf{4}}$ for the mass, momentum, For the turbulent kinetic energy and the dissipation rate of the turbulent kinetic energy, the chemical species conservation equations, energy and the pollution equations, the convergence criteria were set to $10^{-6}$.

\section{- $\quad$ Grid Independence Study}

Before numerical simulation begins, a grid independence study had to be conducted to provide an optimum grid resolution. Four mesh sizes have been generated $(19500,39897,101684,159476)$ to assess the computational mesh solution convergence. "Fig. 3," demonstrate the comparison of temperature radial distribution at axial position $(\mathrm{X} / \mathrm{D}=0.8)$. It can be observed form the figure that (101648) provides excellent results

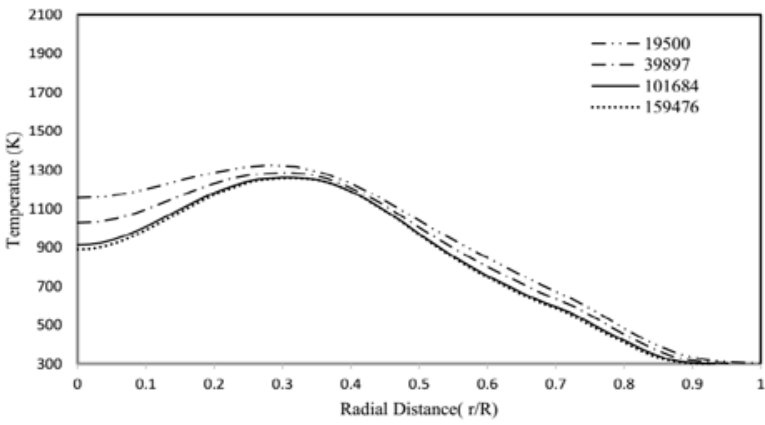

Fig. 3. Radial profile of temperature at station $\mathrm{X} / \mathrm{D}=0.8$

\section{RESULTS AND DISCUSSION}

The results will be discussed in two sections. The first section compares the numerical data, obtained from the RANS models, with the experimental measurements [15], to choose the best model to be implemented for the next section. The second section represents a parametric study of the effect of swirl number and hydrogen addition on combustion characteristics.

\section{A. Comparison of RANS models}

This section compares the numerical data, obtained from the three Turbulence models, with the experimental measurements for SM1 flames. Emphasis is placed on discerning the model's performance of predicting the flow field structures, temperature, mixture fraction and CO mass fraction to choose the best model to be implemented to the study.

Quantitative comparisons of the mean axial velocity components at different axial stations $\mathrm{X} / \mathrm{D}=\{0.136-0.40-$ $0.80-1.2-1.4-2\}$ are depicted in “Fig. 4,”. Observation from the results show that at regions near the inlet stations both the eddy viscosity models and the RSM can predict the axial velocity. Through the rest of the RSM trend show a good agreement to experimental data. The RSM provides 
better performance compared to eddy viscosity models, since its account for stream line curvature and swirling effect.

In "Fig. 5," the comparison of the mean circumferential velocity is indicated, the eddy viscosity models are slightly over- predicted at most location where as the RSM results are quite good. An important feature of the swirl velocity is the Rankine-type vortex (combined forced vortex and a free vortex). This phenomenon can be observed at the middle station $(X / D=0.8)$, the $R S M$ is able to capture such flow features.
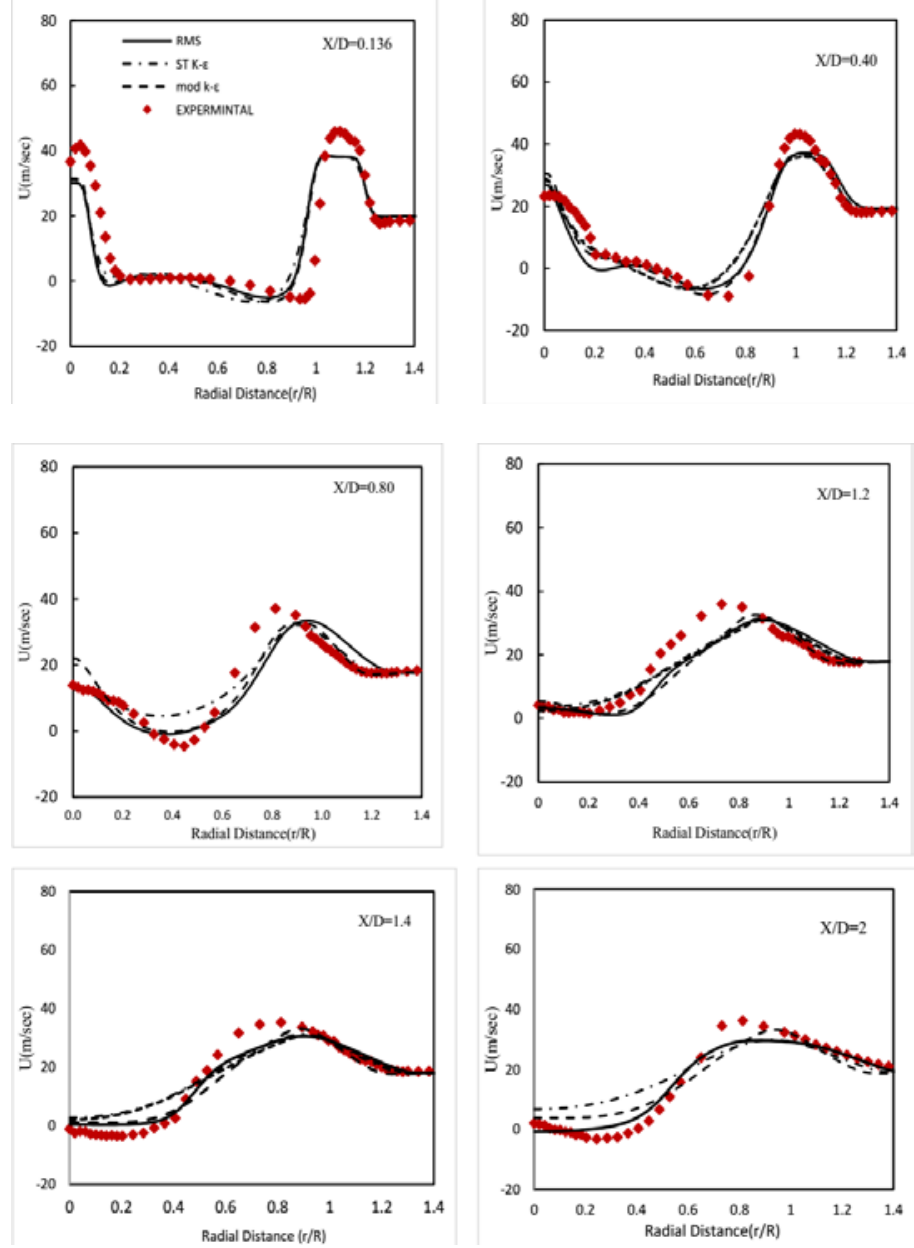

Fig. 4. Radial profile $\mathrm{r} / \mathrm{R}(\mathrm{R}=25 \mathrm{~mm})$ of axial velocity distribution at different axial locations $\mathrm{X} / \mathrm{D}(\mathrm{D}=50 \mathrm{~mm})$.
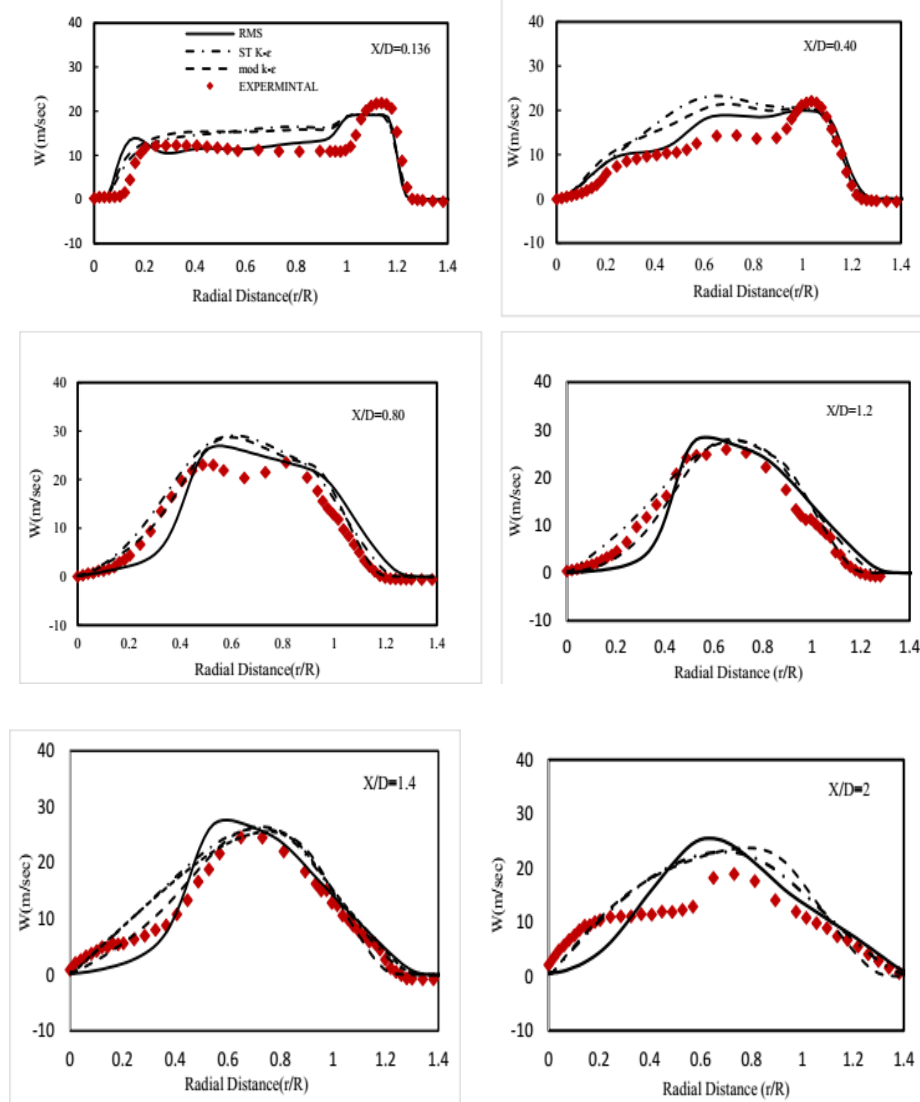

Fig. 5. Radial profile $\mathrm{r} / \mathrm{R}(\mathrm{R}=25 \mathrm{~mm})$ of swirl velocity distribution at different axial locations $\mathrm{X} / \mathrm{D}(\mathrm{D}=50 \mathrm{~mm})$.

Comparisons for the radial profiles of the calculated mean temperatures are presented at different downstream axial locations in "Fig. 6,". It can be observed that the predicted radial profiles of temperature by the RSM are reasonable at most of the axial locations. A noticeable difference in temperature appears between the regions of $\mathrm{x} / \mathrm{D}=0.8$ and $\mathrm{x} / \mathrm{D}=1.1$, where slight under-prediction and over prediction are shown respectively. Other studies that have used different approaches, have reported some discrepancies as well (see Malalasekra et al. [13] and James [14]). Despite the discrepancies mentioned here, the temperature profiles have the same trend as experiments [15]. So, the overall agreement between the calculations obtained by RSM and measurements are good under this case.
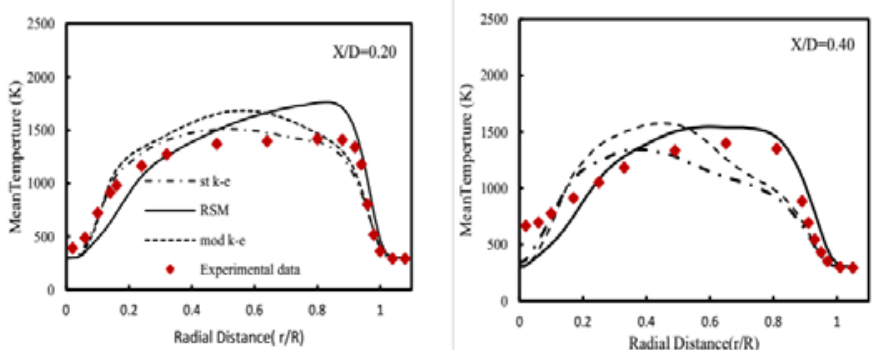

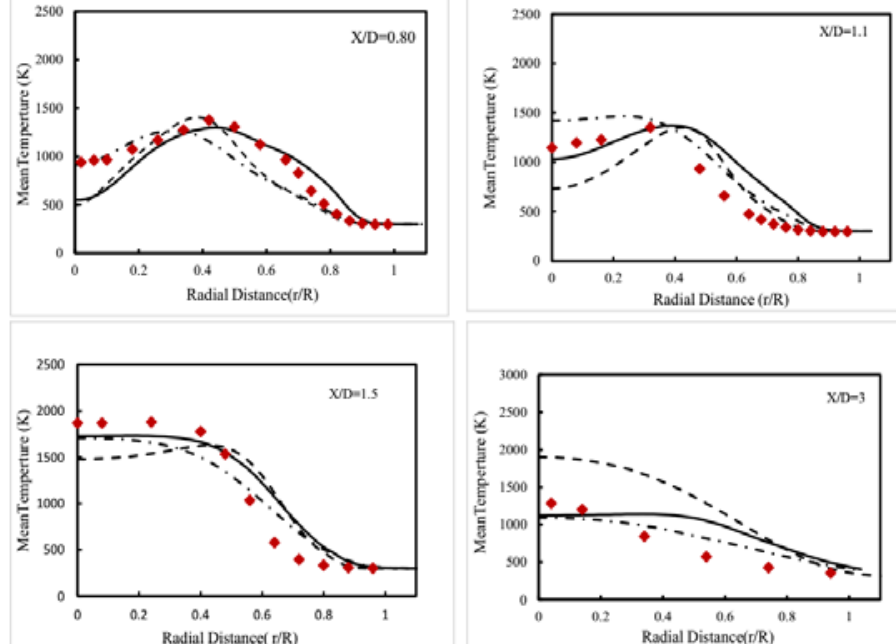

Fig. 6. Radial profile $\mathrm{r} / \mathrm{R}(\mathrm{R}=25 \mathrm{~mm})$ of Mean temperature distribution at different axial locations $\mathrm{X} / \mathrm{D}(\mathrm{D}=50 \mathrm{~mm})$.

The radial profiles of the mean mixture fraction are shown in "Fig. 7,". It can be clearly shown the comparison of the mixture fraction with experimental data is reasonable at most of the axial locations. It appears that there is an over prediction in the Centre line at region of $x / D=0.8$. This can explain why the under prediction for temperature. So, it can be concluded that despite some deviations in predicting the scalar field the overall results of the RSM, together with flamelet model are in a reasonable agreement with experimental data.
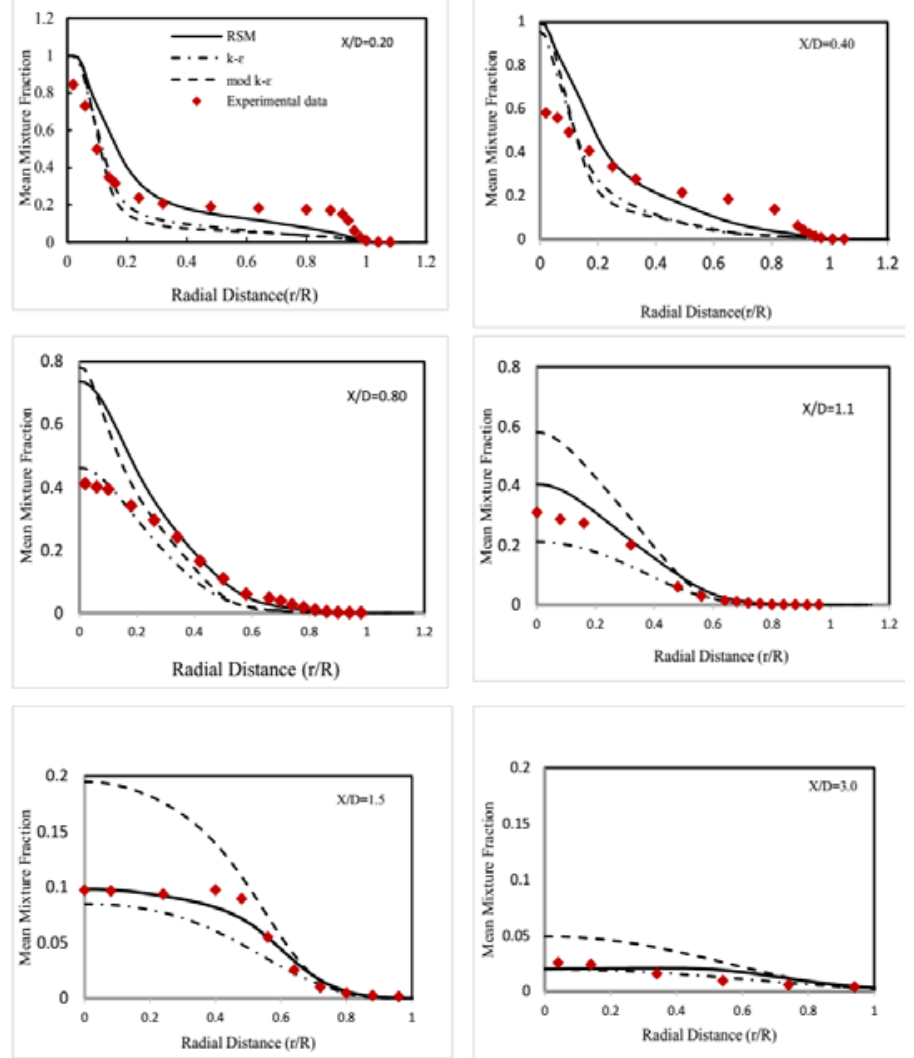

Fig.7. Radial profile $\mathrm{r} / \mathrm{R}(\mathrm{R}=25 \mathrm{~mm})$ of Mean mixture fraction at different axial locations $\mathrm{X} / \mathrm{D}(\mathrm{D}=50 \mathrm{~mm})$.
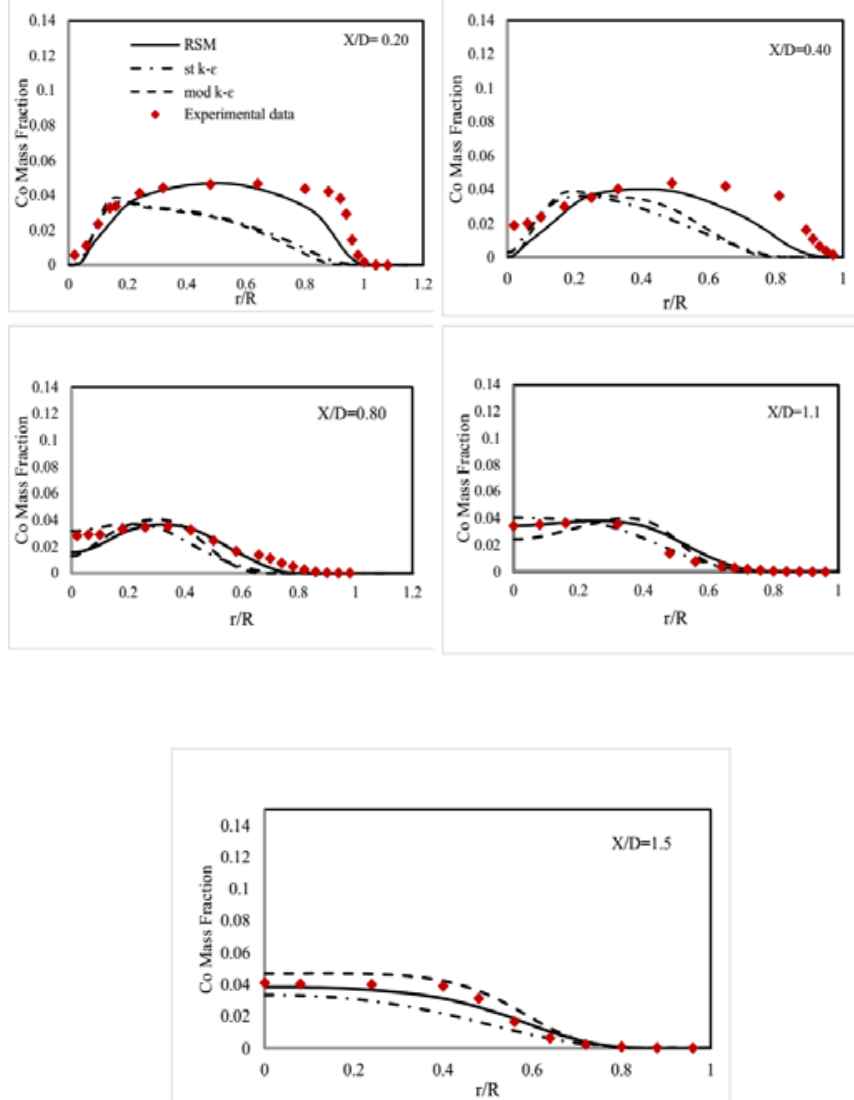

Fig. 8. Radial profile $\mathrm{r} / \mathrm{R}(\mathrm{R}=25 \mathrm{~mm})$ of Mean mass fraction of $\mathrm{CO}$ at different axial locations $\mathrm{X} / \mathrm{D}(\mathrm{D}=50 \mathrm{~mm})$.

The radial distribution of carbon monoxide profiles is depicted in "Fig. 8,". A significant under-prediction is noted in the region close to the bluff-body face for the three turbulence models. Because these values are close to the lean flammability limit of the mixture, a strong impact on carbon monoxide profiles is seen. However, as the flow progresses axially (downstream) the RSM provides an accurate data compared to eddy viscosity models.

An important note that should be mentioned here is that the RSM model coupled with the LFM compared favorably to measurements, previous studies conducted by James et al. [14] show similar findings but with the LES instead of the RSM. This simply implies that for combustion applications, the need for sophisticated numerical techniques are not required, since combustion occurs at the small scales while LES solve the large eddies (energy) and model the small scales. Such indications have also been reported by James. This method is employed for investigation the combustion characteristics for different fuel compositions and swirl numbers.

\section{B. Effect of hydrogen addition on temperature distribution and species concentration}

Combustion characteristics have been investigated by changing the hydrogen concentration in the fuel stream. Flame length is an underlying parameter in the category of diffusion flames by which the structure of the flame could be described reasonably. The axial and radial profiles of the 
calculated mean temperatures are presented at different downstream axial locations in “Fig. 9,". As hydrogen concentration is increased the flame length is decreased. This is due to the decline in the fuel mixture density and reduce the momentum ratio which is defined as:

$$
M R=\frac{\rho_{\text {fuel }}}{\rho_{\text {air }}} \frac{U_{\text {fuel }}^{2}}{U_{\text {air }}^{2}}
$$

This culminates in reduced the penetration of fuel jet which produces more rapid mixing. The effect of hydrogen percent on temperature maps is shown in" Fig. 10,".
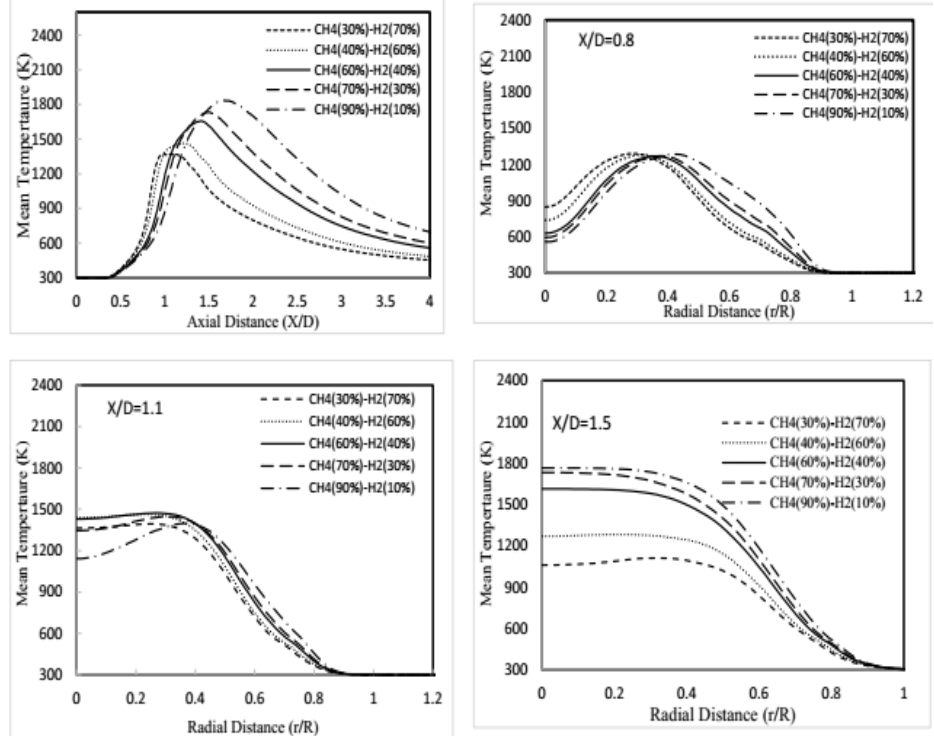

Fig. 9. axial and Radial profile $\mathrm{r} / \mathrm{R}(\mathrm{R}=25 \mathrm{~mm})$ of Mean temperature distribution at different axial locations X/D (D=50mm) for blended CH4$\mathrm{H} 2$

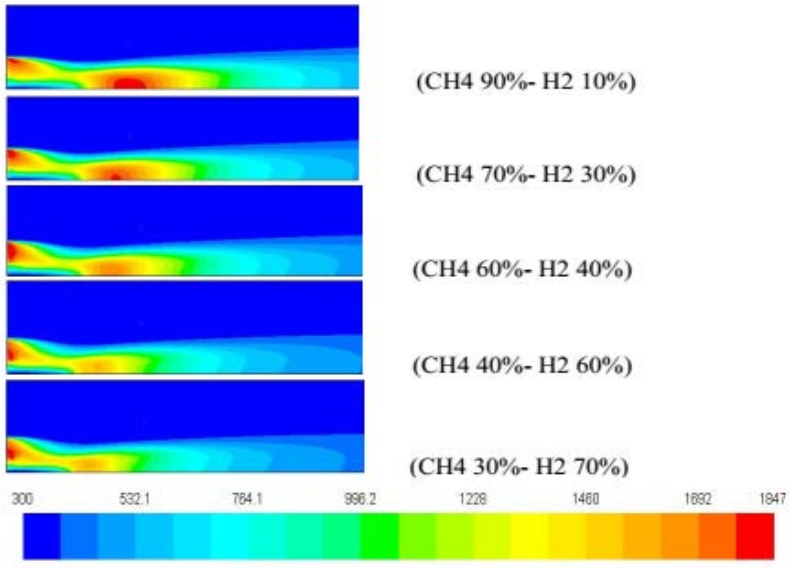

Fig.10. Temperature maps for different mixtures blended CH4-H2

Hydrogen percent has a remarkable effect on $\mathrm{CO}, \mathrm{CO} 2$ species concentrations, "Fig.11," shows the effect of hydrogen addition on axial and radial distribution of $\mathrm{CO}$ and $\mathrm{CO} 2$ species concentrations at different downstream axial locations along the combustor centerline. The increase of hydrogen percentage on fuel results in lower $\mathrm{CO}$ and $\mathrm{CO} 2$ emissions. Th reduction upstream might be ascribed to the reduction in inlet carbon proportion, at downstream the fuel is oxidized to $\mathrm{CO}$ and then to $\mathrm{CO} 2$ and the reduction due to the speed up in CO oxidation.
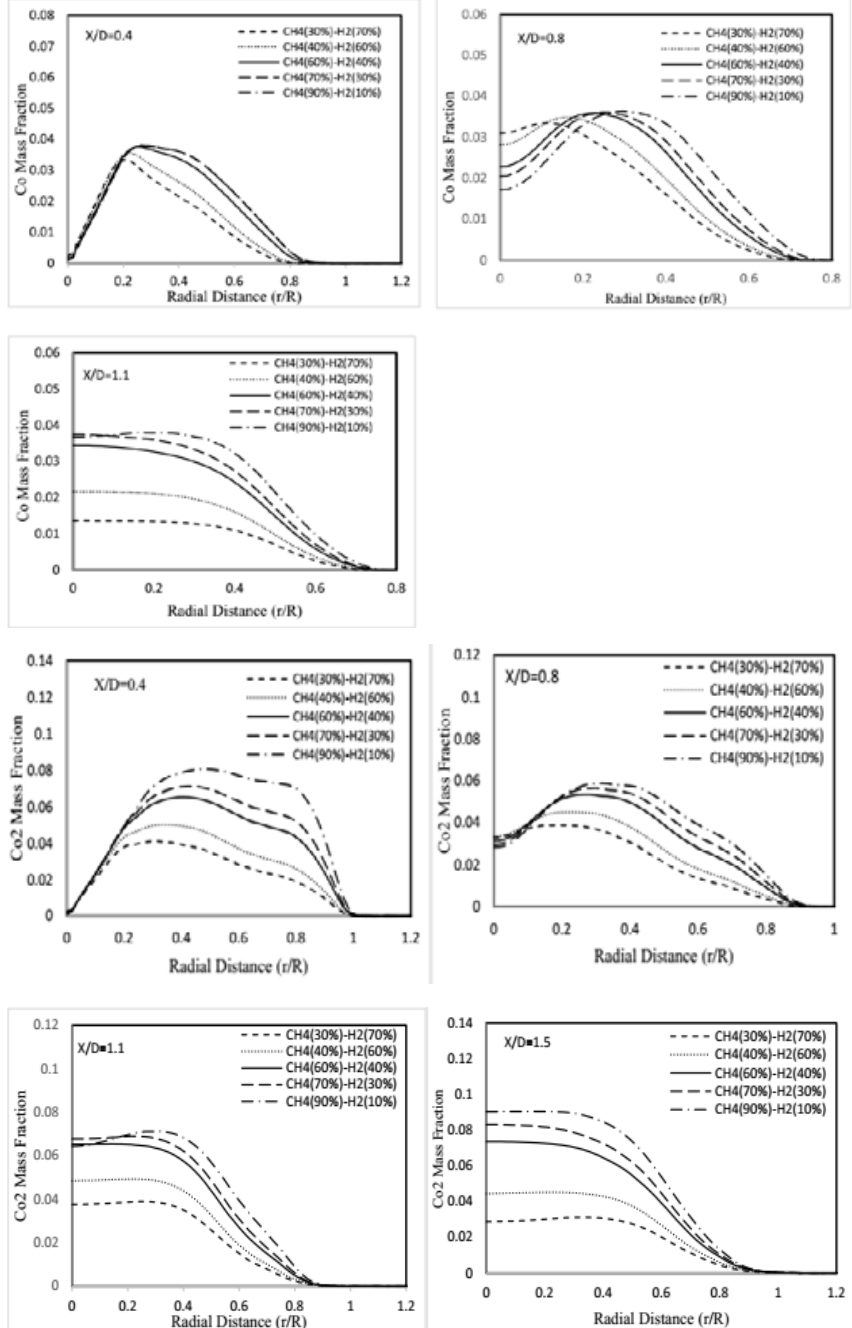

Fig.11. Axial and Radial profile $r / R(R=25 \mathrm{~mm})$ of Mean mass fraction of $\mathrm{CO}$ and $\mathrm{CO} 2$ at different axial locations $\mathrm{X} / \mathrm{D}(\mathrm{D}=50 \mathrm{~mm})$ for blended $\mathrm{CH} 4$

$\mathrm{H} 2$.

\section{Effect of swirl number on temperature distribution and} species concentration

The effect of swirl number on flame temperature gradients along burner axis and radial temperature gradients at different axial distance are illustrated in "Fig. 12,". While the swirl number increase, peak temperature location moves towards bluff body face. The effect of swirl on radial temperature profiles show that as swirl number increased the temperature level decrease because higher swirl number leads to increased mixing between air and fuel streams. 

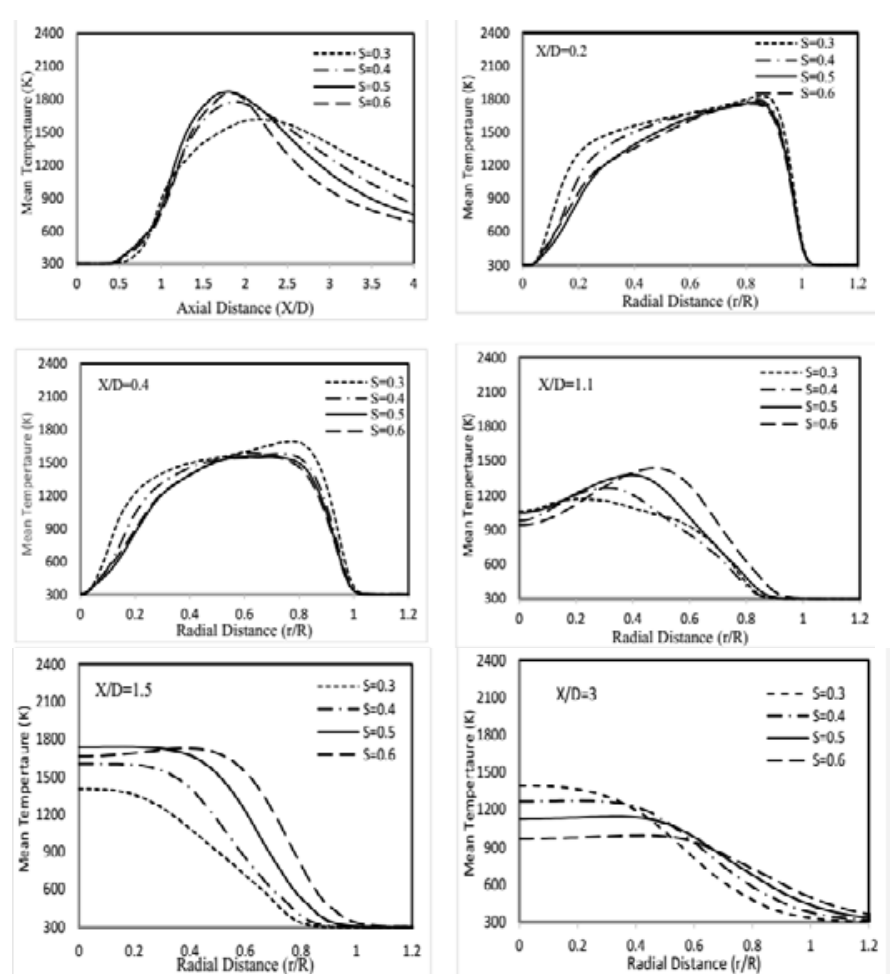

Fig. 12. axial and Radial profile $\mathrm{r} / \mathrm{R}(\mathrm{R}=25 \mathrm{~mm})$ of Mean temperature distribution at different axial locations $X / D(D=50 \mathrm{~mm})$ for distinct swirl number

"Fig. 13," show the effect of swirl number on temperature maps at different swirl numbers, as the swirl number increases, the flame size decreases and the high temperature regions become attached to the upstream direction. Swirl number has an effect on the temperature distribution, since increasing the swirl number leads to the decrease of the flame size and, consequently, to the reduction of the flame temperature inside the combustor, partially at the combustor end. This is a benefit when selecting the turbine blade material. The temperature increase in the front combustor part is due to good mixing between fuel and air, as a result of high turbulence intensity caused by higher swirling effect. Far from the swirler exit, the swirl effect and the turbulence decrease, and this leads to the decrease of the temperature levels.

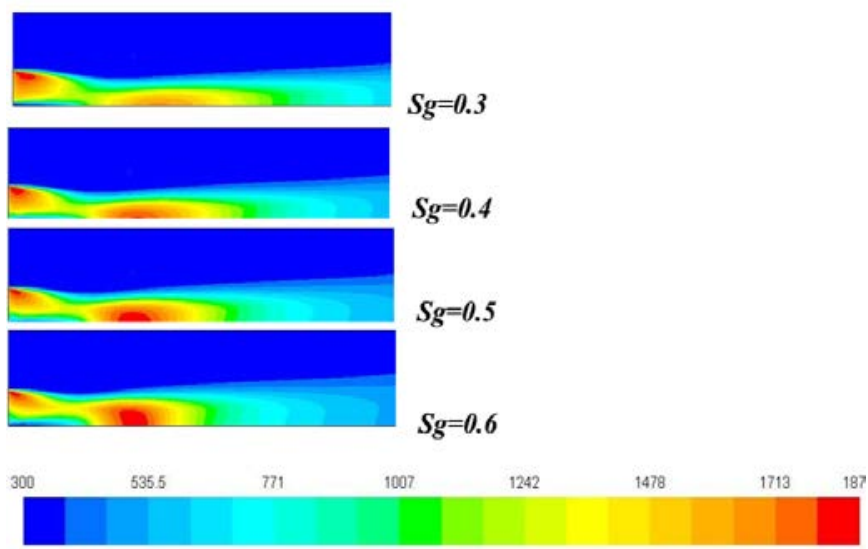

Fig.13. Temperature maps for distinct swirl number

The distribution of $\mathrm{CO}$ and $\mathrm{CO} 2$ along center line of the burner are given for various swirl number are indicated in "Fig. 14,". Both CO and CO2 are the product of chemical combustion so the distribution is directly related to the flame temperature gradient in the same conditions.
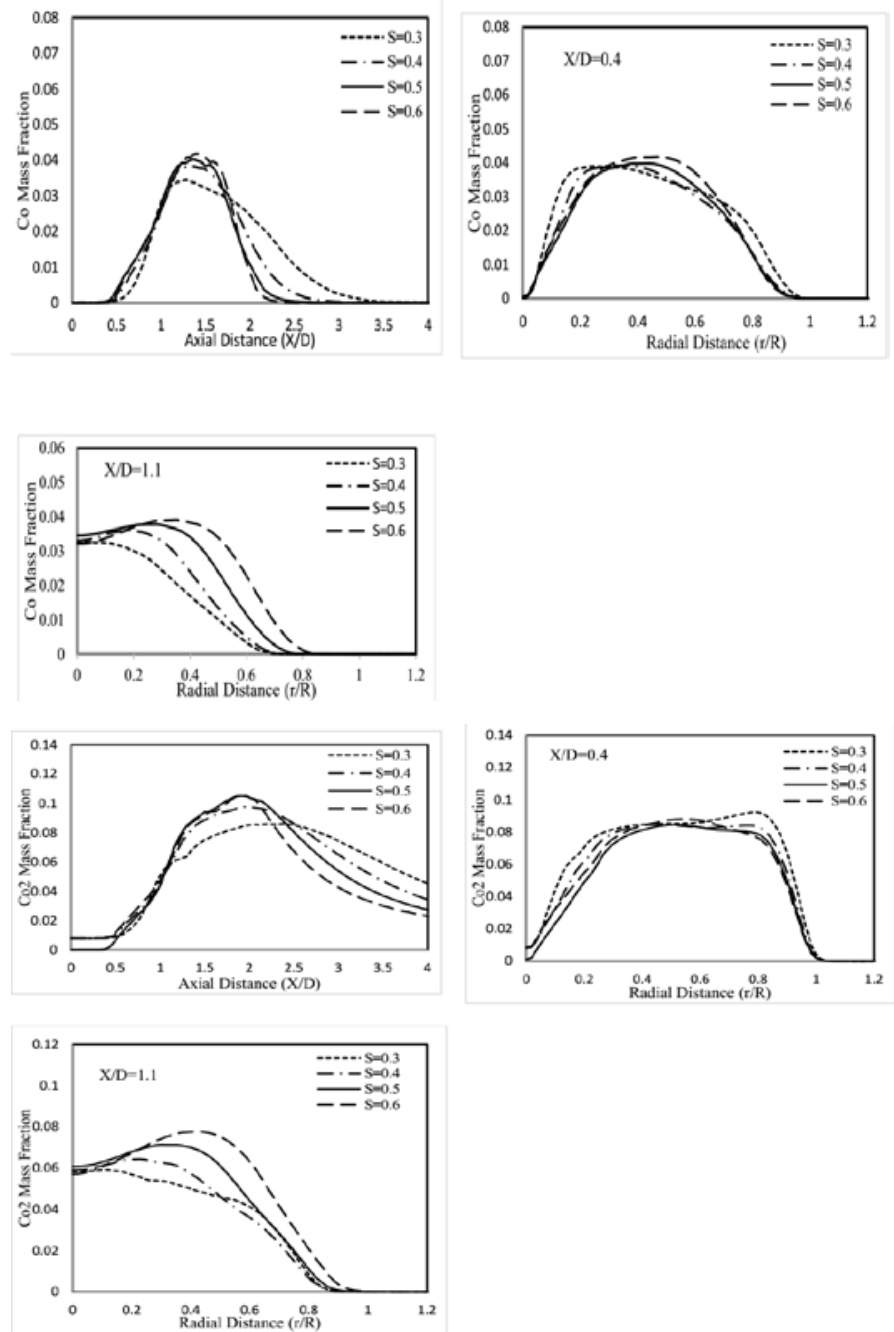

Fig.14. Radial profile $\mathrm{r} / \mathrm{R}(\mathrm{R}=25 \mathrm{~mm})$ of Mean mass fraction of $\mathrm{CO}$ and $\mathrm{CO} 2$ at different axial locations $X / D(D=50 \mathrm{~mm})$ for distinct swirl number. 